\title{
Design and Provisioning of WDM Networks for Many-to-Many Traffic Grooming
}

\author{
Mohammad A. Saleh \\ Ahmed E. Kamal \\ Department of Electrical and Computer Engineering, Iowa State University, Ames, IA 50011
}

\begin{abstract}
A large number of network applications today allow several users to interact together using the many-to-many service mode. A many-to-many session consists of group of users (we refer to them as members), where each member transmits its traffic to all other members in the same group. We address the problem of designing and provisioning of WDM networks to support many-to-many traffic grooming. Our objective is to minimize the overall network cost which is dominated by the cost of transceivers and the number of wavelengths used. We consider three different WDM networks for this problem. One is the non-splitting network, where the nodes do not support optical splitting. The other two networks are the hubbed and the alloptical networks, where the nodes support optical splitting. In the hubbed network, all members in a session transmit their traffic to a designated hub node. Using the new technique of network coding, the hub then linearly combines the traffic units received and sends back to the members a set of linear combinations using light-tree(s). In the all-optical network, each member in a session transmits its traffic directly to all other members in the same session using a light-tree. A comprehensive comparison between the three networks reveals that each of the networks is a cost-effective choice for a certain range of traffic granularities.
\end{abstract}

\section{INTRODUCTION}

Early internet applications such as telnet and ftp are characterized as unicast or "one-to-one". With the advent of the World Wide Web (WWW), a new class of applications has emerged such as video distribution and file distribution which are characterized as multicast or "one-to-many". With more advancements in WWW, another set of applications has emerged such as multimedia conferencing, distance learning, distributed simulations, and collaborative processing [1]. In these applications, each of the participating entities both contributes and receives information to and from the other entities in the same communication session, and therefore are characterized as "many-to-many". In many-to-many communication, a session consists of group of users (we refer to them as members), where each member transmits its traffic to all other members in the same group.

In wavelength routing networks, using wavelength division multiplexing (WDM), it is feasible to have hundreds of wavelengths, each operating at 10 to $40 \mathrm{Gbps}$, per fiber. Bandwidth requirements of user sessions, however, are usually of subwavelength granularities. For example, an MPEG compressed HDTV channel requires less than $20 \mathrm{Mbps}$ of bandwidth. In order to reduce this huge bandwidth gap, traffic grooming was introduced to allow a number of sessions with sub-wavelength granularities to share the bandwidth of a wavelength channel.
Traffic grooming has mainly been considered for unicast traffic [2], [5], [4], [3]. It also has been considered recently for multicast [7], [8] and many-to-one [10] traffic types. For a survey of advances in unicast and multicast traffic grooming, the reader is referred to [6], [9], respectively.

In this paper, we address the problem of designing and provisioning of WDM networks to support many-to-many traffic grooming. This problem can be decomposed into two smaller problems that are not necessarily independent:

- Virtual Topology and Traffic Routing (VTTR) problem, in which we determine what optical channels (lightpaths and light-trees) to establish and how to route and groom each of the subwavelength many-to-many traffic demands on these optical channels.

- Routing and Wavelength Assignment (RWA) problem, in which we determine the route and the wavelength to assign to each of the optical channels on the WDM network.

In our previous work [12], a Mixed Integer Linear Program (MILP) which jointly solves the above two problems was introduced. Although the MILP guaranteed an optimal solution, its complexity was too high. In this paper, our solution approach is to solve each of the VTTR and the $R W A$ problems separately (VTTR then $R W A$ ). This simplifies the overall problem and allows us to obtain efficient solutions. In this work, we also consider three different WDM networks for many-tomany traffic grooming and we provide a comprehensive cost comparison between them.

The major cost of a WDM optical network is dominated by higher layer electronic ports such as IP router ports, MPLS Label Switching Router (LSR) ports and SONET ADM ports (we will refer to these ports as transceivers). A transceiver is needed for each initiation or termination of an optical channel. For example, a lightpath requires two transceivers while a light-tree with $N$ endpoints requires $N$ transceivers. Therefore, our objective in the VTTR problem is to minimize the total number of transceivers $(R)$. In the $R W A$ problem, our objective is to minimize the total number of wavelengths used $(W)$.

\section{A. Assumptions and Notations}

The WDM network has an arbitrary topology represented by an undirected graph $G(V, E)$, with a set of nodes $V$ and a set of physical links $E$, where each physical link $e \in E$ is composed of two unidirectional fibers $\vec{e}$ in opposite directions. The number of wavelengths per fiber is denoted by $W_{F}$, while the grooming factor is denoted by $g$. There 
is a total of $K$ many-to-many session requests, where each session $s_{k}(1 \leq k \leq K)$ has a set of members $m_{s_{k}} \in V$ with cardinality $N_{s_{k}}=\left|m_{s_{k}}\right|$. Each member in $m_{s_{k}}$ has the same traffic demand $t_{s_{k}}$, where $1 \leq t_{s_{k}} \leq g$. We define $H_{s_{k}}=\left\lceil\left(N_{s_{k}}-1\right) t_{s_{k}} / g\right\rceil$ to be a lower bound on the number of incoming channels to a member in a session $s_{k}$ in order to receive the traffic from the other $N_{s_{k}}-1$ members in the same session. Finally, we require that the $t_{s_{k}}$ traffic units originating from a member must not be split among different routes on the virtual topology.

\section{B. The proposed WDM networks}

Designing WDM networks for many-to-many traffic grooming is greatly influenced by whether optical nodes have optical splitting capabilities or not. The following are the WDM networks that we consider for many-to-many traffic grooming (In Section II, we provide a detailed description of each of these networks).

1) Non-Splitting WDM (NSWDM) Network: In this network, nodes do not have optical splitting capabilities and therefore it supports only lightpaths. It will be shown that this network is cost-effective for traffic granularities that are relatively low (e.g., less than one quarter of the capacity of an optical channel).

2) Hubbed WDM (HWDM) Network: In this network, nodes have optical splitting capabilities and therefore it supports lightpaths and light-trees. Each many-to-many session has a designated hub node chosen from one of its members. All the members besides the hub transmit their traffic to the hub through direct lightpaths (upstream traffic). Using the new technique of network coding [13], the hub then linearly combines the traffic units received together with its own traffic units to generate a set of linear combinations. These combinations are then groomed and sent back to the members using direct light-tree(s) (downstream traffic), see Fig. 2.(a) page 4 . It will be shown that this network is cost-effective for traffic granularities that are around half of the capacity of an optical channel.

3) All-Optical WDM (AOWDM) Network: In this network, nodes have optical splitting capabilities. Each member in a many-to-many session transmits it traffic directly to all other members in the same session using a light-tree. Note that no traffic grooming is performed in this network. It will be shown that this network is cost-effective for traffic granularities that are close to the full capacity of an optical channel.

\section{Virtual Topology and TrafFic Routing PRoblem}

\section{A. Non-Splitting WDM Network}

In a NSWDM network, a lightpath (that may span multiple links) can be established between any two nodes in the network. A lightpath may groom traffic from different sessions and traffic from different members within the same session.

Definition 1. A transparent lightpath cycle (TLC) for a manyto-many session $s_{k}$ is a simple cycle of $N_{s_{k}}$ lightpaths that visits each member in $m_{s_{k}}$ exactly once.

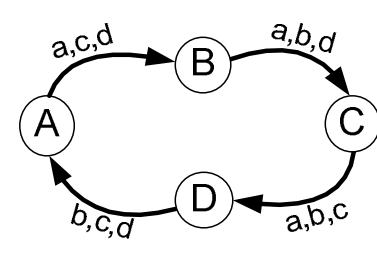

(a)

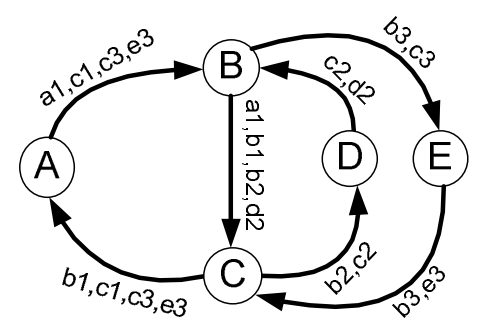

(b)
Fig. 1. (a): TLC for a session $s_{k}$ where $m_{s_{k}}=\{A, B, C, D\}$ each with traffic denoted as $a, b, c$ and $d$, respectively $\left(H_{s_{k}}=1\right)$. (b): Optimal provisioning of sessions $s_{1}, s_{2}$ and $s_{3}$ where $m_{s_{1}}=\{A, B, C\}$ each with one traffic unit denoted as $a 1, b 1, c 1, m_{s_{2}}=\{B, C, D\}$ each with one traffic unit denoted as $b 2, c 2, d 2$, and $m_{s_{3}}=\{B, C, E\}$ each with one traffic unit denoted as $b 3, c 3, e 3(g=4)$.

An example of a $T L C$ for a session $s_{k}$ is shown in Fig. 1.(a). Note that a $T L C$ for $s_{k}$ only describes a virtual topology and it always contains $N_{s_{k}}$ lightpaths regardless of the order of the members and regardless of the underlying physical topology. $T L C s$ serve as an optimal virtual topology for a single session, as indicated by the following theorem:

Theorem 1. An optimal virtual topology that minimizes the total number of transceivers required to provision a single many-to-many session $s_{k}$ in a NSWDM network consists of $H_{s_{k}}$ TLCs for $s_{k}$.

Proof: Any feasible virtual topology to provision $s_{k}$ must at least have a total of $N_{s_{k}} H_{s_{k}}$ lightpaths. This is due to the fact that each member in $m_{s_{k}}$ must at least have $H_{s_{k}}$ lightpaths incoming to receive its traffic. Note that $H_{s_{k}} T L C s$ for $s_{k}$ have exactly $N_{s_{k}} H_{s_{k}}$ lightpaths. Therefore, if we prove it is a feasible virtual topology then it will also be an optimal one. Now, by letting each member to transmit its traffic in the $H_{s_{k}}$ TLCs until it reaches the member just before it in the cycle (see Figure 1.(a)), we guarantee two things. First, exactly $\left(N_{s_{k}}-1\right) t_{s_{k}}$ traffic units are groomed between each pair of consecutive members in the cycle and therefore $H_{s_{k}}$ lightpaths are sufficient to groom this traffic. Second, each member in $m_{s_{k}}$ receives the traffic from all the other members in $m_{s_{k}}$. Therefore, $H_{s_{k}}$ TLCs for $s_{k}$ is a feasible virtual topology.

In the case of multiple sessions, the VTTR problem is generally considered a hard problem due to the correlation between the sessions and the possibility of grooming traffic from different sessions on the same lightpath. Next, we formulate the VTTR problem in a NSWDM network as an Integer Linear Program (ILP).

1) ILP Formulation: The following are the decision variables used in the ILP:

$$
\begin{aligned}
& L_{i j}: \quad \text { number of lightpaths from node } i \text { to node } j(i \neq j) \\
& Z_{i j}^{s k}, p, q \text { : binary number to indicate whether or not the traffic stream }
\end{aligned}
$$




$$
\begin{aligned}
& \text { Minimize } \sum_{i \in V} \sum_{j \in V} L_{i j} \text {. } \\
& \text { Subject to: }
\end{aligned}
$$

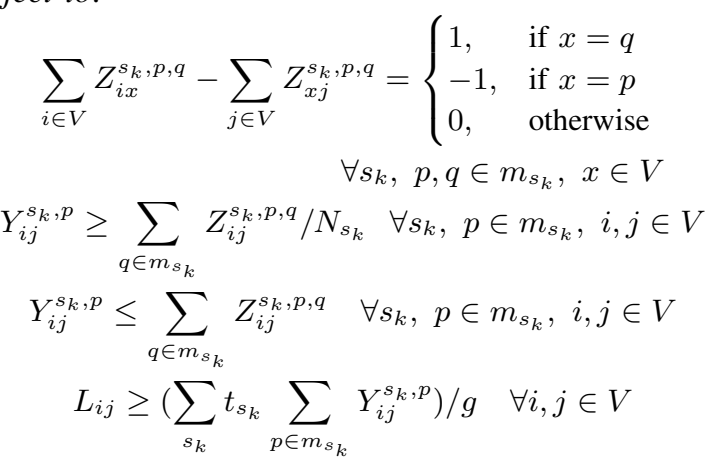

The objective is to minimize the total number of lightpaths or transceivers. Constraint (1) is the flow routing constraint between each pair of members (in both directions) in a manyto-many session. Constraints (2)-(3) set the variable $Y_{i j}^{s_{k}, p}$ as the logical disjunction of all the variables $Z_{i j}^{s_{k}, p, q}$ for all values of $q \in m_{s_{k}}, q \neq p$. Finally, constraint (4) computes the total number of lightpaths needed between each pair of nodes in the network. Note that the ILP is completely independent of the physical topology since it only considers the VTTR problem.

2) Heuristic Solution: After careful examination of the ILP results for small sized instances of the problem and for multiple sessions, we have noticed that many-to-many sessions tend to be provisioned through lightpath cycles. Since a lightpath may groom traffic from different sessions and not just traffic from different members within the same session, these lightpath cycles may share lightpaths and may not be transparent (i.e., number of lightpaths in a lightpath cycle for a session $s_{k}$ may be $>N_{s_{k}}$ ). Fig. 1.(b) clarifies this point by illustrating the optimal provisioning of three many-to-many sessions $s_{1}, s_{2}$ and $s_{3}$ on a 5-node network $\{A, B, C, D, E\}$. Note that the $T L C$ for $s_{1}(A-B-C-A)$ and the $T L C$ for $s_{2}$ $(B-C-D-B)$ share lightpath $B \rightarrow C$, while the $T L C$ for $s_{1}$ and the lightpath cycle for $s_{3}(B-E-C-A-B$ which is not transparent) share lightpaths $C \rightarrow A$ and $A \rightarrow B$.

The heuristic we propose is based on an assumption that each many-to-many session $s_{k}$ is provisioned through $H_{s_{k}}$ lightpath cycles where $\left(N_{s_{k}}-1\right) t_{s_{k}}$ traffic units are groomed between each pair of consecutive members in the lightpath cycles. The heuristic also takes the correlation (sharing of lightpaths) between lightpath cycles into account.

Given $K$ many-to-many session requests, the heuristic tries to build a virtual topology to accommodate the $K$ sessions with the minimum number of lightpaths or transceivers. The current virtual topology is represented in the heuristic as a directed graph $V T$ with a set of nodes that includes every node in $G$ that at least has one lightpath incoming or outgoing. Each directed edge from node $i$ to node $j$ in $V T$ corresponds to a lightpath from node $i$ to node $j$ in $G$. Finally, each edge $(i, j)$ in $V T$ has a capacity $c_{i j}$ representing the remaining capacity on the corresponding lightpath from node $i$ to node $j$ in $G$.

The heuristic (shown in Algorithm 1) has two main steps. First, it orders members in each session $s_{k}$ (this will be the
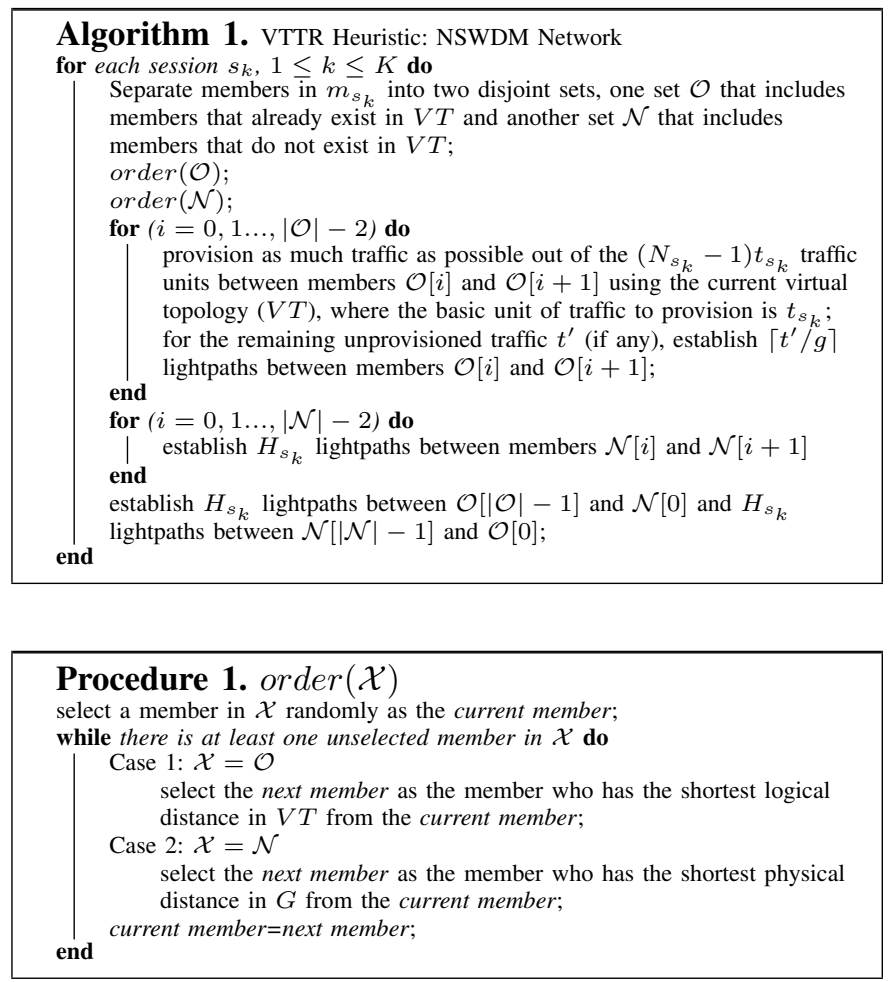

order of the members in the session's $H_{s_{k}}$ lightpath cycles). The way the heuristic orders members in a session $s_{k}$ is by first separating the members in $m_{s_{k}}$ into two disjoint sets $\mathcal{O}$ and $\mathcal{N}$. Afterwards, it orders members in the $\mathcal{O}$ set by minimizing the logical hop distance between each pair of consecutive members, while it orders members in the $\mathcal{N}$ set by minimizing the physical hop distance between each pair of consecutive members (see Procedure 1). The second step of the heuristic is the provisioning of the $\left(N_{s_{k}}-1\right) t_{s_{k}}$ traffic units between each pair of consecutive members in the ordered $m_{s_{k}}$. Between each pair of consecutive members in the $\mathcal{O}$ set, the heuristic attempts to provision as much traffic as possible out of the $\left(N_{s_{k}}-1\right) t_{s_{k}}$ traffic units using the current virtual topology $V T$ without adding new lightpaths. This is done by running a max-flow algorithm between the two members in the current $V T$ (with edge capacities $c_{i j}$ ). For the remaining unprovisioned traffic $t^{\prime}$ (if any), the heuristic establishes $\left\lceil t^{\prime} / g\right\rceil$ lightpaths between the two members. Between each pair of consecutive members in the $\mathcal{N}$ set, the heuristic establishes $H_{s_{k}}$ lightpaths to provision the $\left(N_{s_{k}}-1\right) t_{s_{k}}$ traffic units (after this step, members in $\mathcal{N}$ will be added to $V T$ ). Finally, the heuristic completes the cycle for each session $s_{k}$ by connecting the $\mathcal{O}$ set and the $\mathcal{N}$ set by $H_{s_{k}}$ lightpaths at both ends.

\section{B. Hubbed WDM network}

In a HWDM network, each many-to-many session has a designated hub node chosen from one of its members. All the members besides the hub transmit their $t_{s_{k}}$ traffic units to the hub through direct lightpaths (upstream traffic). Using the new technique of network coding, the hub then linearly combines 
the traffic units received together with its own $t_{s_{k}}$ traffic units to generate $N_{s_{k}}-1$ linearly independent combinations. These combinations must also be linearly independent from the original $t_{s_{k}}$ traffic units received from the members. Afterwards, the $N_{s_{k}}-1$ combinations are groomed and delivered back to the members using direct light-tree(s) (downstream traffic), see Figure 2.(a).

In a HWDM network, each member is guaranteed to recover the original traffic units transmitted by all other members in the same session, as indicated by the following theorem:

Theorem 2. In a HWDM network, each member in a many-tomany session $s_{k}$ will be able to recover the original $t_{s_{k}}$ traffic units transmitted by all other members in the same session.

Proof: In a HWDM network, each member in $m_{s_{k}}$ receives $N_{s_{k}}-1$ linearly independent combinations of the original $t_{s_{k}}$ traffic units transmitted by each member (except the hub which receives the original $t_{s_{k}}$ traffic units directly from the other members). In addition to these combinations, each member has its own $t_{s_{k}}$ traffic units which is also linearly independent from the received combinations. Therefore, each member acquires $N_{s_{k}}$ linearly independent combinations which can be used to solve for the original $t_{s_{k}}$ traffic units.

Note that upstream and downstream traffic stay in the optical domain and optical-electronic-optical $(O / E / O)$ conversion is only performed at the hub. To perform network coding at the hub, we may need to buffer data units that arrive early until all the $N_{s_{k}}-1$ data units arrive. Using Next Generation SONET, multiservice provisioning platform (MSPP) equipments allow up to $128 \mathrm{~ms}$ differential delay between different traffic streams.

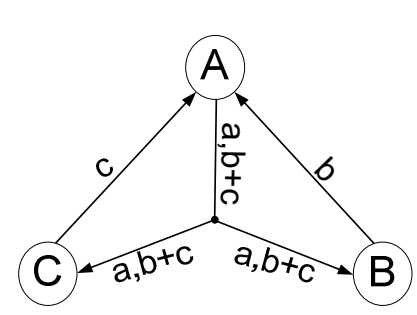

(a)

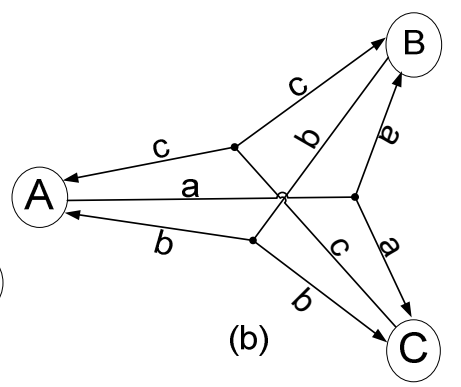

(b)
Fig. 2. Provisioning of a session $s_{k}$ where $m_{s_{k}}=\{A, B, C\}$ each with traffic denoted as $a, b$ and $c$, respectively $\left(H_{s_{k}}=1\right.$ ) in (a): a HWDM network where $H u b\left(s_{k}\right)=A$. (b): an AOWDM network.

According to our previous work [12], light-trees are generally not efficient in grooming traffic from different sessions. Therefore, in this work, we assume that light-trees only groom the linear combinations for the corresponding session. For a single session $s_{k}$, there will be $N_{s_{k}}-1$ upstream lightpaths and $H_{s_{k}}$ downstream light-trees (each with $N_{s_{k}}$ transceivers). In the case of multiple sessions, each session $s_{k}$ still requires $H_{s_{k}}$ downstream light-trees, however, the number of lightpaths depends on the hub selection since a lightpath may groom traffic from different sessions. For example, consider two sessions $s_{1}$ and $s_{2}$ where $m_{s_{1}}=\{A, B, C\}$ and $m_{s_{2}}=\{A, B, D\}, t_{s_{1}}=$

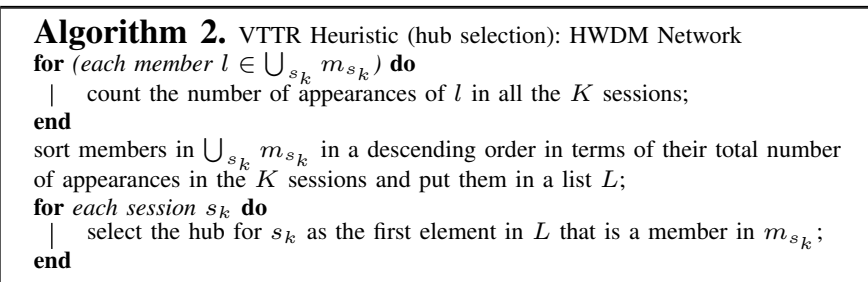

$t_{s_{2}}=1$ and $g=2$. If we select $H u b\left(s_{1}\right)=H u b\left(s_{2}\right)=A$, then there will be a total of 3 upstream lightpaths, $B \rightarrow A$ carrying 2 traffic units (one traffic unit from each session), $C \rightarrow A$ and $D \rightarrow A$ each carrying 1 traffic unit. However, if we select $H u b\left(s_{1}\right)=A$ and $H u b\left(s_{2}\right)=B$ then there will be 4 upstream lightpaths $B \rightarrow A, C \rightarrow A, A \rightarrow B$ and $D \rightarrow B$ each carrying 1 traffic unit. Note that in both cases, each session requires one downstream light-tree. Selecting the hub for each session determines what lightpaths and lighttrees to establish and how we groom traffic from different sessions on the upstream lightpaths, and therefore it solves the VTTR problem. Next, we formulate the VTTR problem (hub selection) in a HWDM network as an ILP.

1) ILP Formulation: We first define $B_{p}^{s_{k}}$ as a binary input parameter that indicates whether or not $p \in m_{s_{k}}$. The following are the decision variables used in ILP:

\footnotetext{
$I_{h}^{s_{k}}: \quad$ binary number to indicate whether or not $h \in m_{s_{k}}$ is the hub node for session $s_{k}$.

$L_{i j}: \quad$ number of lightpaths from node $i$ to node $j(i \neq j)$.
}

$$
\begin{aligned}
& \text { Minimize } \sum_{i \in V} \sum_{j \in V} L_{i j} . \\
& \sum_{h \in m_{s_{k}}} I_{h}^{s_{k}}=1 \quad \forall s_{k} \\
& L_{i j} \geq\left(\sum_{s_{k}} t_{s_{k}} I_{j}^{s_{k}} B_{i}^{s_{k}}\right) / g \quad \forall i, j \in V
\end{aligned}
$$

The objective is to minimize the total number of upstream lightpaths or transceivers. Constraint (5) ensures that there is exactly one hub node for each session chosen from one of its members, while constraint (6) computes the total number of lightpaths needed between each pair of nodes in the network. Note that there is no need to include the downstream direction in the ILP since the number of downstream light-trees is fixed $\left(\sum_{s_{k}} H_{s_{k}}\right)$ and doesn't depend on the hub selection.

2) Heuristic Solution: We now introduce a heuristic approach for the VTTR problem (hub selection) in a HWDM network. The idea of this heuristic is to select the same hub node for as many sessions as possible (see Algorithm 2). The heuristic starts by counting the number of appearances of each member in $\bigcup_{s_{k}} m_{s_{k}}$ in all the $K$ sessions, and then sorts them accordingly in a descending order and put them in a list $L$. Finally, it selects the hub for each session $s_{k}$ as the first element in $L$ that is a member in $m_{s_{k}}$. Selecting the same hub for as many sessions as possible increases the likelihood of inter-session grooming on the upstream direction, which has 
a direct impact on reducing the number of lightpaths needed.

The advantage of network coding in a HWDM network is the reduction of downstream traffic for each session $s_{k}$ from $N_{s_{k}} t_{s_{k}}$ to $\left(N_{s_{k}}-1\right) t_{s_{k}}$ traffic units. Therefore, the total number of transceivers saved ( $R_{\text {saved }}$ ) due to the use of network coding is:

$$
R_{\text {saved }}=\sum_{s_{k}} N_{s_{k}}\left(\left\lceil N_{s_{k}} t_{s_{k}} / g\right\rceil-\left\lceil\left(N_{s_{k}}-1\right) t_{s_{k}} / g\right\rceil\right)
$$

\section{All-Optical WDM network}

In an AOWDM network, each member in a many-to-many session $s_{k}$ transmits it traffic directly to all other members in the same session using a light-tree, see Fig. 2.(b). Note that no traffic grooming is performed and the virtual topology does not depend on $t_{s_{k}}$. Each session $s_{k}$ requires $N_{s_{k}}$ light-trees, while each light-tree requires $N_{s_{k}}$ transceivers. Therefore, the total number of transceivers required is:

$$
R=\sum_{s_{k}} N_{s_{k}}^{2}
$$

\section{Complexity Analysis}

The complexity of the ILPs for NSWDM and HWDM networks in terms of the number of integer variables is $O\left(K|V|^{4}\right)$ and $O\left(|V|^{2}+K|V|\right)$, respectively. The time complexity of Algorithm 1 is $O\left(K|V|^{4}\right)$, while the time complexity of Algorithm 2 is $O\left(K|V|^{2}\right)$.

\section{Routing And WAVELEngth ASSignMENT PROBlem}

Once we solve the VTTR problem and determine the virtual topology, we can then consider the RWA problem. In this problem we need to provision each of the optical channels determined by the VTTR problem on the WDM network by determining: 1) the route of each channel on the network, and 2) the wavelength to assign to each channel, while taking the wavelength continuity constraint into account.

The $R W A$ problem has been extensively studied in the literature and it has been proven to be NP-complete. Many heuristics have been proposed for both the routing and the wavelength assignment problems. For example, fixed routing, fixed-alternate routing, and adaptive routing are some of the well-known approaches for routing, while first fit, least used, and most used are some of the well-known approaches for wavelength assignment. For a review on routing and wavelength assignment approaches, the reader is referred to [11].

Since the $R W A$ problem has been extensively studied, we are only interested in comparing the proposed networks in terms of their consumption of wavelengths. To make the comparison fair and to base it on the merit of the networks only, we use very simple approaches for routing and wavelength assignment. We use shortest path routing and first fit wavelength assignment for lightpaths, while we use shortest path tree routing and first fit wavelength assignment for light-trees.

\section{RESULTS AND COMPARISONS}

In this section, we verify the accuracy of our proposed heuristics and also compare the three networks in terms of the costs $R$ and $W$. For all the experiments we conduct, we set $W_{F}$ large enough to guarantee feasible solutions.

\section{A. Verification of the Heuristics}

To verify the accuracy of our proposed heuristics for NSWDM and HWDM networks, we conduct a number of experiments on small and medium sized networks. Four experiments are conducted on the 6-node network (Net6) shown in Fig. 3.(a), while four other experiments are conducted on the Abilene network [14] shown in Fig. 3.(b). The number of sessions in each experiment is randomly selected between $[3,6]$, while the size of each session is randomly selected between [2,5]. In Net6 experiments, a member in a session is randomly selected between [0,5], while it is randomly selected between $[0,9]$ in the Abilene network experiments. The traffic demand of members in a session, in all the experiments, is randomly selected between $[1,16](g=16)$. Table I compares optimal and heuristic solutions for each of the 8 experiments on each of the two networks (optimal solutions were obtained by solving the corresponding ILP using the CPLEX solver [15]).

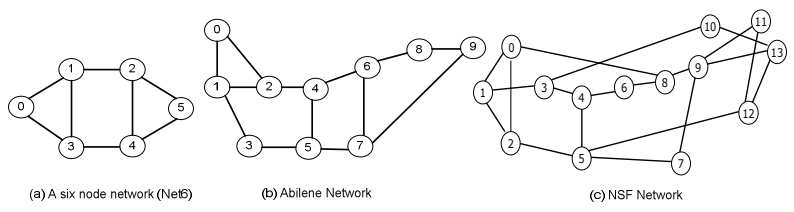

Fig. 3. networks used in the results

TABLE I

NUMBER OF TRANSCEIVERS COMPARISON BETWEEN OPTIMAL AND HEURISTIC APPROACHES FOR NSWDM AND HWDM NETWORKS

\begin{tabular}{c|c|c|c|c|c|c|c|c}
\hline & \multicolumn{4}{|c|}{ Net6 } & \multicolumn{4}{c}{ Abilene } \\
\hline Exp \# & 1 & 2 & 3 & 4 & 5 & 6 & 7 & 8 \\
\hline \hline NSWDM(ILP) & 30 & 22 & 32 & 22 & 36 & 56 & 72 & 36 \\
\hline NSWDM(HEURISTIC) & 32 & 26 & 36 & 24 & 36 & 56 & 76 & 36 \\
\hline \hline HWDM(ILP) & 36 & 27 & 34 & 26 & 36 & 49 & 60 & 33 \\
\hline HWDM(HEURISTIC) & 36 & 29 & 36 & 26 & 40 & 51 & 64 & 35 \\
\hline
\end{tabular}

We can see from the results that solutions from the heuristics either match or are very close to their corresponding optimal solutions.

\section{B. Comparisons}

In this subsection, the three networks will be compared in terms of the costs $R$ and $W$. Since the grooming capabilities of the networks are greatly varied, their performance will be dependent on traffic granularities of sessions in the network. Therefore, we should compare them for different traffic granularities. To make this comparison, we assume a static uniform traffic with all sessions in an experiment having the same traffic demand $t$ (e.g., $t_{s_{1}}=t_{s_{2}}=\ldots=t_{s_{K}}=t$ ), where $1 \leq t \leq g$.

Thirty randomly generated experiments are conducted on the NSF network shown in Fig. 3.(c). The number of sessions in each experiment is randomly selected between $[10,15]$. The 


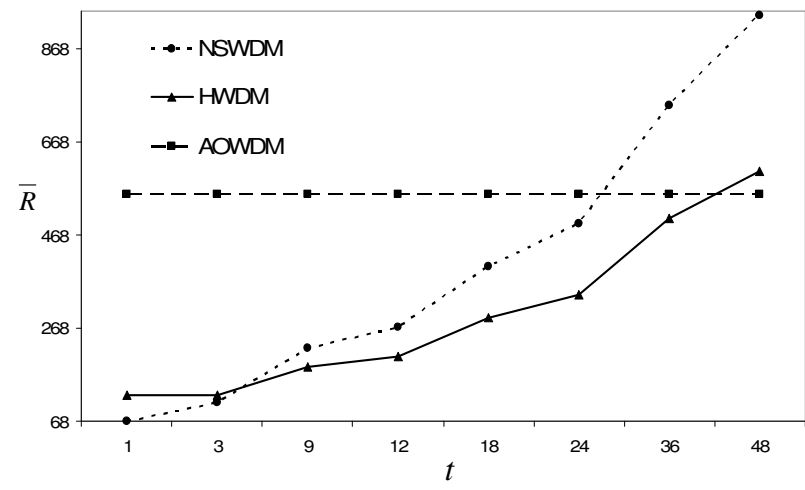

Fig. 4. Values of $\bar{R}$ for $t=\{1,3,9,12,18,24,36,48\}$ on NSF for $g=48$

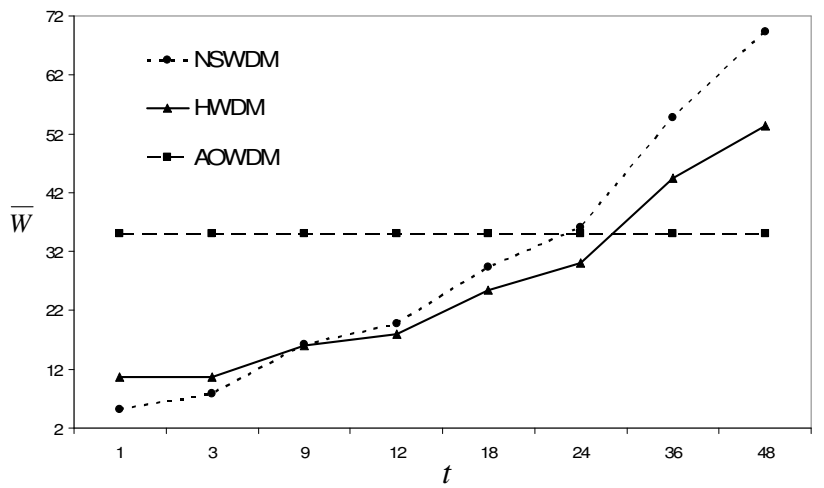

Fig. 5. Values of $\bar{W}$ for $t=\{1,3,9,12,18,24,36,48\}$ on NSF for $g=48$

size of each session is randomly selected between $[2,14]$, while a member in a session is randomly selected between $[0,13]$. Assuming the static uniform traffic, each of the 30 experiments is conducted for all values of $t=\{1,3,9,12,18,24,36,48\}$ $(g=48)$ on all the three networks. These values of $t$ represent the recommended rates for OC streams. Values of $R$ for NSWDM and HWDM networks are obtained by solving the corresponding heuristic. We define $\bar{R}$ to be the average value of all $R$ values obtained from the 30 experiments at a particular value of $t$ on a certain network. The resulting values of $\bar{R}$ are shown in Fig. 4.

After determining the optical channels for each experiment at each value of $t$ on each network, these channels are routed using fixed routing and assigned a wavelength according to first fit scheme (see Section III for details). We also define $\bar{W}$ to be the average value of all $W$ values obtained from the 30 experiments at a particular value of $t$ on a certain network. The resulting values of $\bar{W}$ are shown in Fig. 5 .

From Figs. 4 and 5, we draw the following conclusions:

1) In terms of the cost R: HWDM networks are a costeffective choice for almost three quarters of the spectrum $(g / 8 \leq t \leq 7 g / 8)$. NSWDM networks, on the other hand, are a cost-effective choice for low traffic granularities $(1 \leq t<$ $g / 8)$, while AOWDM networks are a cost-effective choice for high traffic granularities $(7 g / 8<t \leq g)$.

2) In terms of the cost $W$ : NSWDM networks are a costeffective choice for low traffic granularities $(1 \leq t<g / 4)$. HWDM networks, on the other hand, are a cost-effective
TABLE II

VALUES OF $\overline{R_{\text {saved }}}$ AND $\overline{R_{\text {saved }}} / \bar{R}$ FROM THE NSF EXPERIMENTS

\begin{tabular}{c|c|c|c|c|c|c|c|c}
\hline$t$ & 1 & 3 & 9 & 12 & 18 & 24 & 36 & 48 \\
\hline$\overline{R_{\text {saved }}}$ & 0.03 & 0.03 & 12.3 & 13.9 & 23.4 & 32.3 & 49.6 & 73.8 \\
\hline$R_{\text {saved }} / \bar{R}$ & $0 \%$ & $0 \%$ & $6.6 \%$ & $6.7 \%$ & $8 \%$ & $9.5 \%$ & $9.8 \%$ & $14.8 \%$ \\
\hline
\end{tabular}

choice for traffic granularities that lie in the middle $(g / 4 \leq t \leq$ $3 g / 5$ ), while AOWDM networks are a cost-effective choice for high traffic granularities $(t>3 g / 5)$.

Finally, Table II illustrates the advantage of network coding in HWDM networks by showing the values of $\overline{R_{\text {saved }}}$, which is defined as the average value of all $R_{\text {saved }}$ values obtained from the $30 \mathrm{NSF}$ experiments at a particular value of $t$ on the HWDM network. The table also shows the corresponding percentage savings $\left(\overline{R_{\text {saved }}} / \bar{R}\right)$.

\section{CONCLUSIONS}

We have considered three different WDM networks for many-to-many traffic grooming. For each network, we have derived an optimal as well as a heuristic approach to solve the VTTR problem (except for the AOWDM network, where the optimal solution was straightforward). Through extensive experiments, we have concluded that each of the networks is a cost-effective choice (in terms of the costs $R$ and $W$ ) for a certain range of traffic granularities. We have also illustrated the advantage of network coding in reducing the number of transceivers in HWDM networks.

\section{REFERENCES}

[1] B. Quinn and K. Almeroth, "IP multicast applications: challenges and solutions," IETF Request for Comments (RFC) 3170, Sept. 2001.

[2] O. Gerstel, R. Ramaswami, and G. Sasaki, "Cost-effective Traffic Grooming in WDM Rings," IEEE/ACM Transactions on Networking, Oct. 2000.

[3] H. Zhu, H. Zang, K. Zhu, and B. Mukherjee,"A novel generic graph model for traffic grooming in heterogeneous WDM mesh networks," IEEE/ACM Trans. Networking, April 2003.

[4] A. L. Chiu and E. H. Modiano, "Traffic grooming algorithms for reducing electronic multiplexing costs in WDM ring networks" IEEE J. Lightwave Technol. vol. 18, no. 1, pp. 2-12, Jan. 2000.

[5] X. Zhang and C. Qiao,"An effective and comprehensive approach for traffic grooming and wavelength assignment in SONET/WDM rings," IEEE/ACM Trans. Networking, vol. 8, no. 5, pp. 608-617, Oct. 2000.

[6] R. Dutta and G. N. Rouskas,"Traffic grooming in WDM networks: past and future," IEEE Network, vol. 16, no. 6, pp. 46-56, Nov./Dec. 2002.

[7] R. Ul-Mustafa and A. E. Kamal,"Design and provisioning of WDM networks with multicast traffic grooming," IEEE J. Select. Areas Commun., vol. 24 , no. 4, pp. 37-53, Apr. 2006

[8] H. Madhyastha et al., "Grooming of multicast sessions in metropolitan WDM ring networks," Computer Networks, vol. 49, pp. 561-579, 2005.

[9] A. E. Kamal,"Algorithms for Multicast Traffic Grooming in WDM Mesh Networks," IEEE Communications pp. 96-105, Nov. 2006.

[10] R. Ul-Mustafa and A. E. Kamal,"Many-to-one Traffic Grooming with Aggregation in WDM Networks "IEEE J. Select. Areas Commun., vol. 24, pp. 68-81, 2006.

[11] H. Zang, J. P. Jue, and B. Mukherjee,"A review of routing and wavelength assignment approaches for wavelength-routed optical WDM networks," Optical Network Mag., vol. 1, no. 1, pp. 47-60, Jan. 2000.

[12] M. Saleh and A. Kamal, "Many-to-Many Traffic Grooming in WDM Mesh Networks," in proc. IEEE Globecom'08.

[13] R. Ahlswede, N. Cai, S. R. Li, and R. W. Yeung,"Network information flow" IEEE Trans. Inf. Theory vol. 46, no. 4, pp. 1204-1216, 2000.

[14] The Abilene Research Network, http://abilene.internet2.edu/.

[15] http://www.ilog.com/products/cplex/ 